\title{
Associations between trunk-to-peripheral fat ratio and cardiometabolic risk factors in elderly Japanese men: baseline data from the Fujiwara-kyo Osteoporosis Risk in Men (FORMEN) study
}

\author{
Katsuyasu Kouda ${ }^{1 *}$ (D), Yuki Fujita², Kumiko Ohara², Takahiro Tachiki ${ }^{3}$, Junko Tamaki ${ }^{4}$, Akiko Yura²,
} Jong-Seong Moon ${ }^{5}$, Etsuko Kajita ${ }^{3}$, Kazuhiro Uenishi ${ }^{6}$ and Masayuki $1 \mathrm{ki}^{2}$

\begin{abstract}
Background: Body mass-independent parameters might be more appropriate for assessing cardiometabolic abnormalities than weight-dependent indices in Asians who have relatively high visceral adiposity but low body fat. Dual-energy X-ray absorptiometry (DXA)-measured trunk-to-peripheral fat ratio is one such body mass-independent index. However, there are no reports on relationships between DXA-measured regional fat ratio and cardiometabolic risk factors targeting elderly Asian men.

Methods: We analyzed cross-sectional data of 597 elderly men who participated in the baseline survey of the Fujiwara-kyo Osteoporosis Risk in Men (FORMEN) study, a community-based single-center prospective cohort study conducted in Japan. Whole-body fat and regional fat were measured with a DXA scanner. Trunk-to-appendicular fat ratio (TAR) was calculated as trunk fat divided by appendicular fat (sum of arm and leg fat), and trunk-to-leg fat ratio (TLR) as trunk fat divided by leg fat.

Results: Both TAR and TLR in the group of men who used $\geq 1$ medication for hypertension, dyslipidemia, or diabetes ("user group"; $N=347)$ were significantly larger than those who did not use such medication ("non-user group"; $N=250)(P<$ 0.05). After adjusting for potential confounding factors including whole-body fat, both TAR and TLR were significantly associated with low-density lipoprotein cholesterol, high-density lipoprotein cholesterol, triglyceride, fasting serum insulin, and the insulin resistance index in the non-user group and non-overweight men in the non-user group $(N=199)$.
\end{abstract}

Conclusion: The trunk-to-peripheral fat ratio was associated with cardiometabolic risk factors independently of whole-body fat mass. Parameters of the fat ratio may be useful for assessing cardiometabolic risk factors, particularly in underweight to normal-weight populations.

Keywords: Body fat distribution, Densitometry, Epidemiology, Risk factors

\footnotetext{
* Correspondence: koudakat@hirakata.kmu.ac.jp

'Department of Hygiene and Public Health, Kansai Medical University, 2-5-1

Shin-machi, Hirakata, Osaka 573-1010, Japan

Full list of author information is available at the end of the article
}

C C The Author(s). 2021 Open Access This article is licensed under a Creative Commons Attribution 4.0 International License, which permits use, sharing, adaptation, distribution and reproduction in any medium or format, as long as you give appropriate credit to the original author(s) and the source, provide a link to the Creative Commons licence, and indicate if changes were made. The images or other third party material in this article are included in the article's Creative Commons licence, unless indicated otherwise in a credit line to the material. If material is not included in the article's Creative Commons licence and your intended use is not permitted by statutory regulation or exceeds the permitted use, you will need to obtain permission directly from the copyright holder. To view a copy of this licence, visit http://creativecommons.org/licenses/by/4.0/. The Creative Commons Public Domain Dedication waiver (http://creativecommons.org/publicdomain/zero/1.0/) applies to the data made available in this article, unless otherwise stated in a credit line to the data. 


\section{Background}

Some individuals are not obese or overweight according to standard weight tables, but have metabolic abnormalities that are characteristically associated with obesity [1]. Such metabolically obese, normal-weight (MONW) individuals are very common in the general population $[2,3]$. Most MONW individuals have increased abdominal adipose tissue, i.e., a metabolically adverse condition, with a relatively low body mass index (BMI) [4]. Data from the Obesity in Asia Collaboration showed that the absolute risk of diabetes or hypertension tended to be higher among Asians compared with Caucasians for any given level of BMI [5]. Associations between BMI and health risks differ in Asians compared with Europeans. For instance, Asians have a higher prevalence of type 2 diabetes but a lower BMI than Europeans [6]. One possible reason for this difference is that, for any given BMI, Asians have more visceral adiposity compared to Caucasians, and this difference may contribute to lipotoxicity and insulin resistance [7]. In fact, ethnic differences in visceral adiposity as measured by computed tomography have been reported [4].

Waist-to-hip ratio (WHR), a conventional weightindependent parameter and an index of abdominal fat distribution [8], was suggested to be a more appropriate index of cardiometabolic abnormalities than BMI for Asians [6]. Dual-energy X-ray absorptiometry (DXA)-measured trunkto-peripheral fat ratio (a specific surrogate for visceral fat proportion) [9] is also a weight-independent index and a more precise method for measuring abdominal fat distribution than WHR [10]. Asians have been reported to have a higher DXA-measured trunk-to-peripheral fat ratio compared with non-Asians [11].

Associations between DXA-measured truncal fat proportion and cardiometabolic risk factors have also been reported for Europeans [12-14] and North Americans $[15,16]$. However, only limited data are available on the relationship between DXA-measured truncal fat proportion and cardiometabolic risk factors in Asian adults, although a relationship between trunk-to-peripheral fat ratio and blood pressure in children has been reported [17]. Only one report from the Japanese PopulationBased Osteoporosis (JPOS) Cohort Study has reported that DXA-measured trunk-to-peripheral fat ratio in women was associated with cardiometabolic variables [18]. However, no study has reported on associations between DXA-measured truncal fat proportion and cardiometabolic risk factors in elderly Asian men.

The present study aimed to investigate the relationship between DXA-measured trunk-to-peripheral fat ratio and cardiometabolic risk factors in community-dwelling elderly Japanese men using baseline data from the second Fujiwara-kyo Osteoporosis Risk in Men (FORMEN) study.

\section{Methods}

\section{Study population}

The FORMEN study is a large-scale community-based single-center prospective cohort study which aims to determine risk factors for osteoporotic fractures, prevent frailty, increase the number of healthy life years, and enhance the functioning and quality of life of elderly men in Nara Prefecture, Japan. Participants at the baseline survey were aged $\geq 65$ years, lived at home, were able to walk without assistance from another person, and were able to provide self-reported information and written informed consent. The baseline survey of the first cohort of the FORMEN study was conducted from June 2007 to October 2008; details of the first cohort have been described elsewhere [19]. In the present study, the study population consisted of 599 elderly men who participated in the baseline survey of the second cohort, which was conducted from August 2019 to January 2020. After excluding 2 men with missing values for DXA-measured regional fat or blood test, the baseline data of 597 participants were subjected to cross-sectional analysis.

\section{Measurement of whole-body and regional fat}

Whole-body and regional fat mass were measured with a single DXA scanner (QDR-4500A; Hologic Inc., Bedford, MA, USA) mounted on a mobile examination car in the same way as described previously $[17,18]$. The same experienced medical radiology technician performed scans for all participants. Quality control checks of the DXA scanner were completed on a regular basis using several Hologic phantoms. Participants were asked to change into a gown for the scan, remove all metal objects (jewelry, zipper, belts, snaps, underwire bras, etc.) and their shoes, lay flat on the scanner table without a pillow in the dorsal position, and remain still and breath normally during the scan. After the scan, we obtained a whole-body posterior-anterior (PA) scan image of each participant. Arm, leg, and head regions in the whole-body PA scan image were isolated from the trunk region using the following standard manufacturer-recommended methods [10]: (a) the head region: the horizontal neck (shoulder) line just below the chin, (b) arm regions: the vertical shoulder line bisecting the shoulder joints, and (c) leg regions: the lower pelvic divider lines (two angled lines) bisecting both femoral necks. Ratios of trunk-to-peripheral fat were calculated using fat mass volume in the trunk, arm, and leg regions. Trunkto-appendicular fat ratio (TAR) was calculated as trunk fat divided by appendicular fat (sum of arm and leg fat), and trunk-to-leg fat ratio (TLR) as trunk fat divided by leg fat.

\section{Measurements of body size and blood pressure}

Body weight, height, and waist circumference were measured in light clothing with no shoes. BMI was calculated as weight divided by height squared $\left(\mathrm{kg} / \mathrm{m}^{2}\right)$. Overweight and underweight participants were identified 
using BMI cut-offs of $25 \mathrm{~kg} / \mathrm{m}^{2}$ and $18.5 \mathrm{~kg} / \mathrm{m}^{2}$, respectively. Systolic blood pressure and diastolic blood pressure were measured using an automatic oscillometric sphygmomanometer (BP-203i, OMRON COLIN, Tokyo, Japan) with an appropriate cuff size based on the upper arm circumference of each participant. Participants were relaxed and seated with legs uncrossed at an appropriate ambient temperature. Measurements were performed with the right arm supported at the level of the heart after resting for $5 \mathrm{~min}$. The mean value of two readings was used for analysis.

\section{Lifestyle factors and medical history}

Trained health care nurses interviewed participants based on answers to a self-administered questionnaire that covered lifestyle factors including smoking (current

Table 1 Participant characteristics in the user group (use of one or more medication) and the non-user group

\begin{tabular}{|c|c|c|c|}
\hline & \multicolumn{3}{|c|}{ Medication for hypertension, dyslipidemia, or diabetes } \\
\hline & User, $N=347$ & Non-user, $N=250$ & $P$ \\
\hline Age (years) & $74.0 \pm 5.4$ & $72.4 \pm 5.2$ & $<0.01$ \\
\hline Height (cm) & $164.6 \pm 6.0$ & $165.2 \pm 5.6$ & ns \\
\hline Weight (kg) & $64.4 \pm 8.6$ & $61.9 \pm 8.3$ & $<0.01$ \\
\hline Body mass index $\left(\mathrm{kg} / \mathrm{m}^{2}\right)$ & $23.7 \pm 2.7$ & $22.7 \pm 2.7$ & $<0.01$ \\
\hline Overweight, $N(\%)$ & $100(29)$ & $51(20)$ & 0.02 \\
\hline Underweight, $N(\%)$ & $6(2)$ & $18(7)$ & $<0.01$ \\
\hline Waist circumference $(\mathrm{cm})$ & $86.9 \pm 7.4$ & $83.8 \pm 7.9$ & $<0.01$ \\
\hline Whole-body fat (kg) & $13.9 \pm 4.7$ & $11.8 \pm 4.4$ & $<0.01$ \\
\hline Trunk fat (kg) & $7.4 \pm 3.0$ & $6.1 \pm 2.8$ & $<0.01$ \\
\hline Arm fat (kg) & $1.4 \pm 0.5$ & $1.3 \pm 0.5$ & $<0.01$ \\
\hline Leg fat (kg) & $4.2 \pm 1.5$ & $3.8 \pm 1.4$ & $<0.01$ \\
\hline TAR & $1.31 \pm 0.37$ & $1.19 \pm 0.36$ & $<0.01$ \\
\hline TLR & $1.79 \pm 0.57$ & $1.61 \pm 0.54$ & $<0.01$ \\
\hline MET-minutes/week, median (25, 75\%ile) & $2106(1025,4176)$ & $2523(1067,4718)$ & ns \\
\hline Current smoker, $N(\%)$ & $32(9)$ & $24(10)$ & ns \\
\hline Alcohol intake (kcal/day) & $86 \pm 131$ & $97 \pm 146$ & ns \\
\hline Energy intake (kcal/day) & $1693 \pm 320$ & $1716 \pm 296$ & ns \\
\hline $\mathrm{NaCl}$ intake (g/day) & $10.0 \pm 1.8$ & $10.1 \pm 1.9$ & ns \\
\hline Systolic blood pressure $(\mathrm{mmHg})$ & $138.9 \pm 16.9$ & $139.5 \pm 18.8$ & ns \\
\hline Diastolic blood pressure (mmHg) & $77.5 \pm 9.9$ & $80.5 \pm 10.5$ & $<0.01$ \\
\hline LDL cholesterol (mg/dl) & $111.3 \times / \div 1.3$ & $119.4 \times 1 \div 1.3$ & $<0.01$ \\
\hline HDL cholesterol (mg/dl) & $59.8 \times / \div 1.3$ & $63.6^{\times} / \div 1.3$ & $<0.01$ \\
\hline Total cholesterol (mg/dl) & $196.8 \times / \div 1.2$ & $209.6 \times 1 \div 1.2$ & $<0.01$ \\
\hline Triglyceride (mg/dl) & $100.7^{\times /} \div 1.6$ & $92.0 \times / \div 1.6$ & 0.02 \\
\hline Hemoglobin A1c (\%) & $6.0 \times / \div 1.1$ & $5.7 \times / \div 1.1$ & $<0.01$ \\
\hline Fasting serum insulin (mU/l) & $4.8 \times / \div 2.0$ & $3.5 \times / \div 1.8$ & $<0.01$ \\
\hline Fasting plasma glucose (mg/dl) & $106.1 \times / \div 1.3$ & $98.0 \times / \div 1.2$ & $<0.01$ \\
\hline HOMA-IR & $1.3 \times / \div 2.4$ & $0.9 \times / \div 2.0$ & $<0.01$ \\
\hline Aspartate aminotransferase (U/l) & $24.3 \times / \div 1.3$ & $24.1 \times / \div 1.3$ & ns \\
\hline Alanine aminotransferase $(\mathrm{U} / \mathrm{I})$ & $19.7 \times / \div 1.5$ & $19.1 \times / \div 1.4$ & ns \\
\hline Estimated glomerular filtration rate $\left(\mathrm{ml} / \mathrm{min} / 1.73 \mathrm{~m}^{2}\right)$ & $64.9 \pm 14.3$ & $69.9 \pm 13.2$ & $<0.01$ \\
\hline
\end{tabular}

Data are expressed as mean \pm SD, median (25,75\%ile), geometric mean $\times / \div$ SD, or $N(\%)$

Biochemical marker values were logarithmically converted and then statistically analyzed

$P$ values were calculated using the unpaired $t$ test or Mann-Whitney $U$ test

$P<0.05$ was considered statistically significant

MET-minutes/week was calculated using the International Physical Activity Questionnaire

$N$, number; ns, not significant; TAR, trunk-to-appendicular fat ratio; TLR, trunk-to-leg fat ratio; MET, metabolic equivalent of task; HDL, high-density lipoprotein; LDL, low-density lipoprotein; HOMA-IR, homeostasis model assessment-insulin resistance; SD, standard deviation 
Table 2 Differences between user and non-user groups according to the type of medication

\begin{tabular}{|c|c|c|c|c|c|c|c|c|c|}
\hline & \multicolumn{9}{|c|}{ Medication for } \\
\hline & \multicolumn{3}{|c|}{ Hypertension } & \multicolumn{3}{|l|}{ Dyslipidemia } & \multicolumn{3}{|l|}{ Diabetes } \\
\hline & $\begin{array}{l}\text { User, } \\
N=256\end{array}$ & $\begin{array}{l}\text { Non-user, } \\
N=341\end{array}$ & $P$ & $\begin{array}{l}\text { User, } \\
N=167\end{array}$ & $\begin{array}{l}\text { Non-user, } \\
N=430\end{array}$ & $P$ & $\begin{array}{l}\text { User, } \\
n=92\end{array}$ & $\begin{array}{l}\text { Non-user, } \\
N=505\end{array}$ & $P$ \\
\hline Age (years) & $74.7 \pm 5.5$ & $72.3 \pm 5.1$ & $\begin{array}{l}< \\
0.01\end{array}$ & $73.9 \pm 5.6$ & $73.1 \pm 5.3$ & ns & $73.6 \pm 4.6$ & $73.3 \pm 5.5$ & ns \\
\hline Height (cm) & $164.0 \pm 5.7$ & $165.4 \pm 5.9$ & $\begin{array}{l}< \\
0.01\end{array}$ & $165.0 \pm 6.4$ & $164.8 \pm 5.6$ & ns & $164.9 \pm 5.7$ & $164.8 \pm 5.9$ & ns \\
\hline Weight (kg) & $64.5 \pm 8.9$ & $62.5 \pm 8.2$ & $\begin{array}{l}< \\
0.01\end{array}$ & $64.7 \pm 8.5$ & $62.8 \pm 8.5$ & 0.02 & $64.2 \pm 9.0$ & $63.2 \pm 8.5$ & ns \\
\hline Body mass index $\left(\mathrm{kg} / \mathrm{m}^{2}\right)$ & $23.9 \pm 2.7$ & $22.8 \pm 2.7$ & $\begin{array}{l}< \\
0.01\end{array}$ & $23.7 \pm 2.4$ & $23.1 \pm 2.8$ & 0.01 & $23.6 \pm 3.0$ & $23.2 \pm 2.7$ & ns \\
\hline Overweight, N (\%) & $79(31)$ & $72(21)$ & $\begin{array}{l}< \\
0.01\end{array}$ & $46(28)$ & $105(24)$ & ns & $24(26)$ & $127(25)$ & ns \\
\hline Underweight, $N(\%)$ & $3(1)$ & $21(6)$ & $\begin{array}{l}< \\
0.01\end{array}$ & $3(2)$ & $21(5)$ & ns & $3(3)$ & $21(4)$ & ns \\
\hline Waist circumference $(\mathrm{cm})$ & $87.3 \pm 7.4$ & $84.3 \pm 7.8$ & $\begin{array}{l}< \\
0.01\end{array}$ & $87.0 \pm 7.0$ & $85.1 \pm 8.0$ & $\begin{array}{l}< \\
0.01\end{array}$ & $86.8 \pm 7.9$ & $85.4 \pm 7.7$ & ns \\
\hline Whole-body fat (kg) & $14.3 \pm 4.6$ & $12.1 \pm 4.6$ & $\begin{array}{l}< \\
0.01\end{array}$ & $14.0 \pm 4.5$ & $12.6 \pm 4.7$ & $\begin{array}{l}< \\
0.01\end{array}$ & $13.0 \pm 5.1$ & $13.0 \pm 4.6$ & ns \\
\hline Trunk fat (kg) & $7.7 \pm 2.9$ & $6.2 \pm 2.9$ & $\begin{array}{l}< \\
0.01\end{array}$ & $7.5 \pm 2.8$ & $6.6 \pm 3.0$ & $\begin{array}{l}< \\
0.01\end{array}$ & $7.0 \pm 3.1$ & $6.8 \pm 2.9$ & ns \\
\hline Arm fat (kg) & $1.5 \pm 0.6$ & $1.3 \pm 0.5$ & $\begin{array}{l}< \\
0.01\end{array}$ & $1.5 \pm 0.5$ & $1.3 \pm 0.5$ & $\begin{array}{l}< \\
0.01\end{array}$ & $1.4 \pm 0.6$ & $1.4 \pm 0.5$ & ns \\
\hline Leg fat (kg) & $4.3 \pm 1.5$ & $3.8 \pm 1.4$ & $\begin{array}{l}< \\
0.01\end{array}$ & $4.2 \pm 1.5$ & $4.0 \pm 1.5$ & 0.03 & $3.9 \pm 1.6$ & $4.1 \pm 1.5$ & ns \\
\hline TAR & $1.34 \pm 0.37$ & $1.21 \pm 0.36$ & $\begin{array}{l}< \\
0.01\end{array}$ & $1.34 \pm 0.36$ & $1.23 \pm 0.37$ & $\begin{array}{l}< \\
0.01\end{array}$ & $1.35 \pm 0.42$ & $1.25 \pm 0.36$ & 0.02 \\
\hline TLR & $1.83 \pm 0.58$ & $1.63 \pm 0.54$ & $\begin{array}{l}< \\
0.01\end{array}$ & $1.83 \pm 0.56$ & $1.67 \pm 0.56$ & $\begin{array}{l}< \\
0.01\end{array}$ & $1.85 \pm 0.65$ & $1.69 \pm 0.54$ & 0.01 \\
\hline $\begin{array}{l}\text { MET-minutes/week, } \\
\text { median ( } 25,75 \% \text { ile) }\end{array}$ & $\begin{array}{l}2118(1188 \\
3801)\end{array}$ & $\begin{array}{l}2502(990 \\
4764)\end{array}$ & ns & $\begin{array}{l}2070(1097 \\
3792)\end{array}$ & $\begin{array}{l}2337(1017, \\
4632)\end{array}$ & ns & $\begin{array}{l}1855(1066 \\
3660)\end{array}$ & $\begin{array}{l}2376(1032 \\
4590)\end{array}$ & ns \\
\hline Current smoker, $N(\%)$ & $23(9)$ & $33(10)$ & ns & $13(8)$ & $43(10)$ & ns & $12(13)$ & $44(9)$ & ns \\
\hline Alcohol intake (kcal/day) & $92 \pm 133$ & $90 \pm 141$ & ns & $73 \pm 111$ & $97 \pm 145$ & 0.03 & $62 \pm 93$ & $96 \pm 143$ & $\begin{array}{l}< \\
0.01\end{array}$ \\
\hline Energy intake (kcal/day) & $1681 \pm 330$ & $1718 \pm 295$ & ns & $1679 \pm 293$ & $1712 \pm 317$ & ns & $1699 \pm 350$ & $1703 \pm 303$ & ns \\
\hline $\mathrm{NaCl}$ intake (g/day) & $10.1 \pm 1.8$ & $10.0 \pm 1.9$ & ns & $10.0 \pm 1.8$ & $10.0 \pm 1.9$ & ns & $10.1 \pm 1.8$ & $10.0 \pm 1.9$ & ns \\
\hline $\begin{array}{l}\text { Systolic blood pressure } \\
(\mathrm{mmHg})\end{array}$ & $140.0 \pm 16.9$ & $138.5 \pm 18.2$ & ns & $138.8 \pm 17.4$ & $139.3 \pm 17.8$ & ns & $134.8 \pm 16.5$ & $139.9 \pm 17.8$ & 0.01 \\
\hline $\begin{array}{l}\text { Diastolic blood pressure } \\
(\mathrm{mmHg})\end{array}$ & $77.8 \pm 10.1$ & $79.5 \pm 10.4$ & 0.04 & $77.4 \pm 10.0$ & $79.3 \pm 10.4$ & 0.04 & $73.5 \pm 9.8$ & $79.7 \pm 10.1$ & $\begin{array}{l}< \\
0.01\end{array}$ \\
\hline LDL cholesterol (mg/dl) & $110.9 \times / \div 1.3$ & $117.5 \times / \div 1.3$ & 0.01 & $107.0 \times / \div 1.3$ & $117.7^{\times /} \div 1.3$ & $\begin{array}{l}< \\
0.01\end{array}$ & $104.3 \times / \div 1.3$ & $116.6^{\times /} \div 1.3$ & $\begin{array}{l}< \\
0.01\end{array}$ \\
\hline HDL cholesterol (mg/dl) & $58.8 \times / \div 1.3$ & $63.4 \times / \div 1.3$ & $\begin{array}{l}< \\
0.01\end{array}$ & $59.1 \times / \div 1.2$ & $62.3 \times / \div 1.3$ & 0.01 & $58.7^{\times} / \div 1.3$ & $61.9 \times / \div 1.3$ & 0.04 \\
\hline Total cholesterol (mg/dl) & $195.6^{\times} / \div 1.2$ & $207.1 \times / \div 1.2$ & $\begin{array}{l}< \\
0.01\end{array}$ & $191.9^{\times} \div 1.2$ & $206.2^{\times} / \div 1.2$ & $\begin{array}{l}< \\
0.01\end{array}$ & $188.1 \times / \div 1.2$ & $204.7^{\times /} \div 1.2$ & $\begin{array}{l}< \\
0.01\end{array}$ \\
\hline Triglyceride (mg/dl) & $101.5 \times / \div 1.6$ & $93.7 \times / \div 1.6$ & 0.04 & $105.2 \times / \div 1.6$ & $93.9^{\times} / \div 1.6$ & $\begin{array}{l}< \\
0.01\end{array}$ & $110.7^{\times} / \div 1.6$ & $94.7^{\times} / \div 1.6$ & $\begin{array}{l}< \\
0.01\end{array}$ \\
\hline Hemoglobin A1c (\%) & $5.9 \times / \div 1.1$ & $5.9 \times / \div 1.1$ & ns & $6.0 \times / \div 1.1$ & $5.8 \times / \div 1.1$ & $\begin{array}{l}< \\
0.01\end{array}$ & $6.8 \times / \div 1.1$ & $5.7^{\times} / \div 1.1$ & $\begin{array}{l}< \\
0.01\end{array}$ \\
\hline $\begin{array}{l}\text { Fasting serum insulin } \\
(\mathrm{mU} / \mathrm{l})\end{array}$ & $4.8^{\times} / \div 2.0$ & $3.8 \times / \div 1.9$ & $\begin{array}{l}< \\
0.01\end{array}$ & $4.9^{\times} / \div 1.9$ & $4.0 \times / \div 2.0$ & $\begin{array}{l}< \\
0.01\end{array}$ & $7.3 \times / \div 2.6$ & $3.8^{\times} / \div 1.8$ & $\begin{array}{l}< \\
0.01\end{array}$ \\
\hline
\end{tabular}

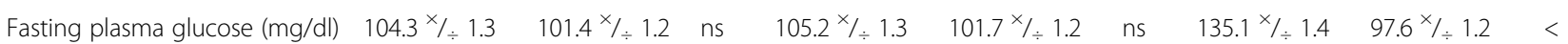


Table 2 Differences between user and non-user groups according to the type of medication (Continued)

\begin{tabular}{|c|c|c|c|c|c|c|c|c|c|}
\hline & \multicolumn{9}{|c|}{ Medication for } \\
\hline & \multicolumn{3}{|l|}{ Hypertension } & \multicolumn{3}{|l|}{ Dyslipidemia } & \multicolumn{3}{|l|}{ Diabetes } \\
\hline & $\begin{array}{l}\text { User, } \\
N=256\end{array}$ & $\begin{array}{l}\text { Non-user, } \\
N=341\end{array}$ & $P$ & $\begin{array}{l}\text { User, } \\
N=167\end{array}$ & $\begin{array}{l}\text { Non-user, } \\
N=430\end{array}$ & $P$ & $\begin{array}{l}\text { User, } \\
n=92\end{array}$ & $\begin{array}{l}\text { Non-user, } \\
N=505\end{array}$ & $P$ \\
\hline & & & & & & & & & 0.01 \\
\hline HOMA-IR & $1.2 \times / \div 2.4$ & $1.0 \times / \div 2.2$ & $\begin{array}{l}< \\
0.01\end{array}$ & $1.3 \times / \div 2.2$ & $1.0 \times / \div 2.3$ & $\begin{array}{l}< \\
0.01\end{array}$ & $2.4 \times / \div 3.2$ & $0.9 \times / \div 1.9$ & $\begin{array}{l}< \\
0.01\end{array}$ \\
\hline $\begin{array}{l}\text { Aspartate aminotransferase } \\
(\mathrm{U} / \mathrm{l})\end{array}$ & $24.1 \times / \div 1.3$ & $24.3 \times / \div 1.3$ & ns & $24.6^{\times} / \div 1.3$ & $24.0 \times / \div 1.3$ & ns & $23.0 \times / \div 1.4$ & $24.4 \times / \div 1.3$ & 0.04 \\
\hline $\begin{array}{l}\text { Alanine aminotransferase } \\
(\mathrm{U} / \mathrm{I})\end{array}$ & $19.4 \times / \div 1.5$ & $19.4 \times / \div 1.4$ & ns & $20.1 \times / \div 1.5$ & $19.2 \times / \div 1.5$ & ns & $20.0 \times / \div 1.5$ & $19.3^{\times} / \div 1.4$ & ns \\
\hline $\begin{array}{l}\text { Estimated glomerular } \\
\text { filtration rate }\left(\mathrm{ml} / \mathrm{min} / 1.73 \mathrm{~m}^{2}\right)\end{array}$ & $64.0 \pm 14.6$ & $69.2 \pm 13.3$ & $\begin{array}{c}< \\
0.01\end{array}$ & $62.7 \pm 14.4$ & $68.7 \pm 13.6$ & $\begin{array}{c}< \\
0.01\end{array}$ & $64.7 \pm 16.1$ & $67.4 \pm 13.7$ & ns \\
\hline
\end{tabular}

Data are expressed as mean \pm SD, median (25, 75\%ile), geometric mean $\times / \div$ SD, or $N(\%)$

Biochemical marker values were logarithmically converted and then statistically analyzed

$P$ values were calculated using the unpaired $t$ test or Mann-Whitney $U$ test. $P<0.05$ was considered statistically significant

MET-minutes/week was calculated using the International Physical Activity Questionnaire

$N$, number; ns, not significant; TAR, trunk-to-appendicular fat ratio; TLR, trunk-to-leg fat ratio; MET, metabolic equivalent of task; HDL, high-density lipoprotein; LDL, low-density lipoprotein; HOMA-IR, homeostasis model assessment-insulin resistance; SD, standard deviation

or non-smoker) and physical activity. Participants were asked to bring current prescriptions of medications to the baseline visit, and the nurses recorded the names and doses of the medications. Food frequency questionnaire (FFQ) for dietary intake of nutrients in Japan (FFQ for the Prevention and Management of Osteoporosis; FFQPOP [20]) was used to estimate dietary nutrient intake. Participants were asked to select a grade of frequency of food intake during the past 1 month. Responses to the questionnaire were also verified by dietitians during their interviews with participants. Intakes of nutrients and energy were estimated using the Standard Table of Food Composition in Japan [21]. Information on physical activity was obtained using the Japanese version of the International Physical Activity Questionnaire (IPAQ), which has been validated for Japanese adults aged 65 years and older [22]. Total weekly physical activity (metabolic equivalent of task (MET)-minutes/week) was estimated in accordance with official IPAQ guidelines [23].

\section{Biochemical analysis}

Blood samples were obtained by vein puncture after an overnight fast for conventional biochemical tests. Levels of triglyceride $(\mathrm{mg} / \mathrm{dl})$ were determined by the enzymatic method (glycerol phosphate oxidase with glycerol blank) (Pureauto S TG-N, Sekisui Medical Co., Ltd., Tokyo, Japan); high-density lipoprotein (HDL; mg/dl) cholesterol by the Sekisui HDL direct method (Cholestest N HDL, Sekisui Medical Co., Ltd., Tokyo, Japan); lowdensity lipoprotein (LDL; $\mathrm{mg} / \mathrm{dl}$ ) cholesterol by the Sekisui LDL direct method (Cholestest LDL, Sekisui Medical Co., Ltd., Tokyo, Japan); total cholesterol (mg/dl) by the ultraviolet method with cholesterol dehydrogenase (T-
$\mathrm{CHO}$ reagents, KL and "Kokusai", Sysmex Corp., Kobe, Japan); fasting plasma glucose $(\mathrm{mg} / \mathrm{dl})$ by the ultraviolet method with hexokinase (CicaLiquid GLU, Kanto Chemical Co., Inc., Tokyo, Japan); Hemoglobin A1c (\%, National Glycohemoglobin Standardization Program) by the latex aggregation immunoassay (RAPIDIA Auto HbA1c, Fujirebio Inc., Tokyo, Japan); fasting serum insulin $(\mu \mathrm{U} / \mathrm{ml})$ by the chemiluminescent enzyme immunoassay (Lumipulse Presto Insulin, Fujirebio Inc., Tokyo, Japan); aspartate aminotransferase (U/l) by the Japan Society of Clinical Chemistry recommended method (CicaLiquid AST, Kanto Chemical Co., Inc., Tokyo, Japan); alanine aminotransferase (U/l) by the Japan Society of Clinical Chemistry recommended method (CicaLiquid ALT, Kanto Chemical Co., Inc., Tokyo, Japan); and creatinine $(\mathrm{mg} / \mathrm{dl})$ by the enzymatic method (Determiner L CRE, Hitachi Chemical Diagnostics Systems Co., Ltd., Tokyo Japan). To estimate insulin resistance, homeostasis model assessment-insulin resistance (HOMA-IR) was calculated using fasting plasma glucose and fasting serum insulin [24]. Estimated glomerular filtration rate (eGFR; $\mathrm{ml} / \mathrm{min} / 1.73 \mathrm{~m}^{2}$ ) was calculated using the Modification of Diet in Renal Disease Study modified for Japanese individuals by the Japanese Society of Nephrology as follows: eGFR $=194 \times$ serum creatinine $^{-1.094} \times$ $\operatorname{age}^{-0.287}[25]$.

\section{Statistical analyses}

All statistical analyses were performed with SPSS Statistics Desktop for Japan, Version 22 (IBM Japan, Ltd., Tokyo, Japan). Frequency distributions for the biochemical tests were determined. We confirmed that levels of biochemical markers were distributed log-normally. Therefore, these values were logarithmically converted 
Table 3 Correlations between TAR/TLR and cardiometabolic variables, and between TAR/TLR and potential confounders

\begin{tabular}{|c|c|c|c|c|c|c|c|c|c|c|c|c|c|c|c|c|c|c|c|c|}
\hline & \multicolumn{2}{|l|}{ Age } & \multicolumn{2}{|c|}{ Height } & \multicolumn{2}{|c|}{$\begin{array}{l}\text { MET- } \\
\text { minutes } \\
\text { /week }\end{array}$} & \multicolumn{2}{|c|}{$\begin{array}{l}\text { Current } \\
\text { smoker }\end{array}$} & \multicolumn{2}{|c|}{$\begin{array}{l}\text { Alcohol } \\
\text { intake }\end{array}$} & \multicolumn{2}{|c|}{$\begin{array}{l}\text { Energy } \\
\text { intake }\end{array}$} & \multicolumn{2}{|c|}{$\begin{array}{l}\mathrm{NaCl} \\
\text { intake }\end{array}$} & \multicolumn{2}{|c|}{$\begin{array}{l}\text { Whole- } \\
\text { body fat }\end{array}$} & \multicolumn{2}{|l|}{ TAR } & \multicolumn{2}{|l|}{ TLR } \\
\hline & $r$ & $P$ & $r$ & $P$ & $r$ & $P$ & $r$ & $P$ & $r$ & $P$ & $r$ & $P$ & $r$ & $P$ & $r$ & $P$ & $r$ & $P$ & $r$ & $P$ \\
\hline \multicolumn{21}{|l|}{ Non-user group, $N=250$} \\
\hline Systolic blood pressure & 0.06 & ns & $\overline{-} 0.01$ & ns & 0.15 & 0.01 & 0.03 & ns & 0.14 & 0.03 & $\begin{array}{l}- \\
0.04\end{array}$ & ns & $-\overline{0}$ & ns & 0.04 & ns & 0.04 & ns & 0.04 & ns \\
\hline Diasto & $\begin{array}{l}- \\
0.21\end{array}$ & $\begin{array}{l}< \\
0.01\end{array}$ & 0.14 & 0.03 & 0.07 & ns & 0.05 & ns & 0.11 & ns & 0.03 & ns & $\overline{-} .02$ & ns & 0.15 & 0.02 & 0.07 & ns & 0.06 & ns \\
\hline LDL chole & $\begin{array}{l}- \\
0.16\end{array}$ & 0.01 & 0.11 & ns & $\begin{array}{l}- \\
0.10\end{array}$ & ns & 0.07 & ns & $\begin{array}{l}- \\
0.08\end{array}$ & ns & $\begin{array}{l}- \\
0.01\end{array}$ & ns & $\begin{array}{l}- \\
0.02\end{array}$ & ns & 0.15 & 0.02 & 0.22 & $\begin{array}{l}< \\
0.01\end{array}$ & 0.23 & $\begin{array}{l}< \\
0.01\end{array}$ \\
\hline HDL chole & 0.05 & ns & $-\overline{0}$ & ns & 0.08 & ns & $\begin{array}{l}- \\
0.17\end{array}$ & $\begin{array}{l}< \\
0.01\end{array}$ & 0.22 & $\begin{array}{l}< \\
0.01\end{array}$ & $\begin{array}{l}- \\
0.10\end{array}$ & nc & $-\overline{0}$ & ns & $\begin{array}{l}- \\
0.41\end{array}$ & $\begin{array}{l}< \\
0.01\end{array}$ & $\begin{array}{l}- \\
0.34\end{array}$ & $\begin{array}{l}< \\
0.01\end{array}$ & $\begin{array}{l}- \\
0.31\end{array}$ & $\begin{array}{l}< \\
0.01\end{array}$ \\
\hline Total cholesterol & $\begin{array}{l}- \\
0.14\end{array}$ & 0.02 & 0.09 & ns & $\overline{0} .03$ & ns & 0.01 & ns & 0.02 & ns & $\begin{array}{l}- \\
0.07\end{array}$ & ns & $-\overline{0}$ & ns & 0.04 & ns & 0.13 & 0.04 & 0.15 & 0.02 \\
\hline Trigl & $\begin{array}{l}- \\
0.11\end{array}$ & ns & 0.07 & ns & $\overline{-} 0.02$ & ns & 0.11 & ns & $\begin{array}{l}- \\
0.07\end{array}$ & ns & 0.04 & ns & $-\overline{0}$ & ns & 0.44 & $\begin{array}{l}< \\
0.01\end{array}$ & 0.42 & $\begin{array}{l}< \\
0.01\end{array}$ & 0.42 & $\begin{array}{l}< \\
0.01\end{array}$ \\
\hline Hem & $\begin{array}{l}- \\
0.03\end{array}$ & ns & 0.02 & ns & 0.03 & ns & $\begin{array}{l}- \\
0.03\end{array}$ & ns & $\begin{array}{l}- \\
0.14\end{array}$ & 0.03 & 0.03 & ns & $\overline{-} .03$ & ns & 0.10 & ns & 0.17 & $\begin{array}{l}< \\
0.01\end{array}$ & 0.19 & $\begin{array}{l}< \\
0.01\end{array}$ \\
\hline Fast & $\begin{array}{l}- \\
0.16\end{array}$ & 0.01 & 0.03 & ns & $\begin{array}{l}- \\
0.14\end{array}$ & 0.03 & 0.01 & ns & $\begin{array}{l}- \\
0.09\end{array}$ & $n$ & $\begin{array}{l}- \\
0.02\end{array}$ & n & $-\overline{0.07}$ & ns & 0.49 & $\begin{array}{l}< \\
0.01\end{array}$ & 0.41 & $\begin{array}{l}< \\
0.01\end{array}$ & 0.39 & $\begin{array}{l}< \\
0.01\end{array}$ \\
\hline Fasting plasma glucose & $\overline{-} 0.07$ & ns & $\overline{0} .02$ & ns & $\overline{0} .07$ & ns & 0.00 & ns & 0.01 & ns & $\overline{-} 0.05$ & ns & 0.00 & ns & 0.09 & ns & 0.19 & $\begin{array}{l}< \\
0.01\end{array}$ & 0.18 & $\begin{array}{l}< \\
0.01\end{array}$ \\
\hline HOMA-IR & $\begin{array}{l}- \\
0.16\end{array}$ & 0.01 & 0.02 & ns & $\begin{array}{l}- \\
0.14\end{array}$ & 0.02 & 0.01 & ns & $-\overline{0.08}$ & ns & $\overline{-} .03$ & ns & $-\overline{0}$ & ns & 0.46 & $\begin{array}{l}< \\
0.01\end{array}$ & 0.41 & $\begin{array}{l}< \\
0.01\end{array}$ & 0.39 & $\begin{array}{l}< \\
0.01\end{array}$ \\
\hline $\begin{array}{c}\text { Aspartate } \\
\text { aminotransferase }\end{array}$ & 0.09 & ns & $\begin{array}{c}- \\
0.04\end{array}$ & ns & 0.02 & ns & $-\overline{10}$ & ns & 0.14 & 0.03 & $\overline{0}-$ & 0.04 & $\begin{array}{c}- \\
0.05\end{array}$ & ns & $\overline{0}-$ & 0.05 & 0.06 & ns & 0.07 & $n$ \\
\hline Alanine aminotransferase & $-\overline{11}$ & ns & $\begin{array}{c}- \\
0.05\end{array}$ & ns & 0.02 & ns & 0.01 & ns & 0.07 & ns & 0.01 & ns & $\overline{0 .}$ & 0.03 & 0.11 & ns & 0.23 & $\begin{array}{c}< \\
0.01\end{array}$ & 0.22 & $\begin{array}{c}< \\
0.01\end{array}$ \\
\hline $\begin{array}{l}\text { Estimated glomerular } \\
\text { filtration rate }\end{array}$ & $\overline{0 .}$ & $\begin{array}{c}< \\
0.01\end{array}$ & 0.05 & ns & 0.09 & ns & 0.05 & ns & 0.09 & ns & 0.06 & ns & 0.03 & ns & $\overline{0 .}$ & ns & $\overline{0 .}$ & 0.03 & $\overline{0 .}$ & 0. \\
\hline TAR & $-\overline{0}$ & $\begin{array}{l}< \\
0.01\end{array}$ & 0.00 & ns & $\overline{-}-05$ & ns & 0.03 & ns & 0.06 & ns & $-\overline{0}$ & ns & 0.00 & ns & 0.47 & $\begin{array}{l}< \\
0.01\end{array}$ & N/A & & N/A & \\
\hline TLR & - & $\begin{array}{l}< \\
0.01\end{array}$ & $\overline{0} .01$ & ns & - & ns & 0.01 & ns & 0.06 & ns & $\frac{-}{0.07}$ & ns & 0.01 & ns & 0.43 & $\begin{array}{l}< \\
0.01\end{array}$ & N/A & & N/A & \\
\hline
\end{tabular}

Non-overweight men of the non-user group, $N=199$

\begin{tabular}{|c|c|c|c|c|c|c|c|c|c|c|c|c|c|c|c|c|c|c|c|c|}
\hline Systolic blood pressure & 0.12 & ns & $\begin{array}{l}- \\
0.05\end{array}$ & ns & 0.11 & ns & 0.03 & ns & 0.19 & 0.01 & $\begin{array}{l}- \\
0.09\end{array}$ & ns & $\begin{array}{l}- \\
0.03\end{array}$ & ns & 0.11 & ns & 0.04 & ns & 0.03 & ns \\
\hline Diastolic blood pressure & $\overline{0} .20$ & $\begin{array}{l}< \\
0.01\end{array}$ & 0.12 & ns & 0.06 & ns & 0.06 & ns & 0.15 & 0.04 & 0.00 & ns & $\overline{0} 0.05$ & ns & 0.21 & $\begin{array}{l}< \\
0.01\end{array}$ & 0.05 & ns & 0.04 & ns \\
\hline LDL cholesterol & $\begin{array}{l}- \\
0.13\end{array}$ & ns & 0.15 & 0.03 & $\overline{0} .08$ & ns & 0.07 & ns & $\overline{0}-06$ & ns & 0.01 & ns & $\begin{array}{l}- \\
0.02\end{array}$ & ns & 0.27 & $\begin{array}{l}< \\
0.01\end{array}$ & 0.30 & $\begin{array}{l}< \\
0.01\end{array}$ & 0.30 & $\begin{array}{l}< \\
0.01\end{array}$ \\
\hline HDL cholesterol & 0.07 & ns & $\overline{0.04}$ & ns & 0.08 & ns & $\begin{array}{l}- \\
0.16\end{array}$ & 0.02 & 0.23 & $\begin{array}{l}< \\
0.01\end{array}$ & $\overline{0}-07$ & ns & $\overline{0}-03$ & ns & $\overline{0.38}$ & $\begin{array}{l}< \\
0.01\end{array}$ & $\overline{0} .33$ & $\begin{array}{l}< \\
0.01\end{array}$ & $\overline{0}-30$ & $\begin{array}{l}< \\
0.01\end{array}$ \\
\hline Total cholesterol & $\begin{array}{l}- \\
0.11\end{array}$ & ns & 0.12 & ns & $\begin{array}{l}- \\
0.01\end{array}$ & ns & 0.01 & ns & 0.05 & ns & $\overline{0}-07$ & ns & $\begin{array}{l}- \\
0.07\end{array}$ & ns & 0.18 & 0.01 & 0.21 & $\begin{array}{l}< \\
0.01\end{array}$ & 0.23 & $\begin{array}{l}< \\
0.01\end{array}$ \\
\hline Triglyceride & $\begin{array}{l}- \\
0.10\end{array}$ & ns & 0.08 & ns & 0.00 & ns & 0.11 & ns & $\begin{array}{l}- \\
0.11\end{array}$ & ns & $\begin{array}{l}- \\
0.04\end{array}$ & ns & $\begin{array}{l}- \\
0.07\end{array}$ & ns & 0.43 & $\begin{array}{l}< \\
0.01\end{array}$ & 0.41 & $\begin{array}{l}< \\
0.01\end{array}$ & 0.41 & $\begin{array}{l}< \\
0.01\end{array}$ \\
\hline Hemoglobin A1C & $\overline{0}-03$ & ns & 0.04 & ns & 0.03 & ns & $\overline{-}-02$ & ns & $\begin{array}{l}- \\
0.14\end{array}$ & 0.05 & 0.01 & ns & $\begin{array}{l}- \\
0.05\end{array}$ & ns & 0.06 & ns & 0.13 & ns & 0.13 & ns \\
\hline Fasting serum insulin & $\begin{array}{l}- \\
0.13\end{array}$ & ns & 0.05 & ns & $\begin{array}{l}- \\
0.13\end{array}$ & ns & 0.01 & ns & $\begin{array}{l}- \\
0.14\end{array}$ & ns & $\overline{0}-05$ & ns & $\begin{array}{l}- \\
0.10\end{array}$ & ns & 0.44 & $\begin{array}{l}< \\
0.01\end{array}$ & 0.37 & $\begin{array}{l}< \\
0.01\end{array}$ & 0.33 & $\begin{array}{l}< \\
0.01\end{array}$ \\
\hline Fasting plasma glucose & $\begin{array}{l}- \\
0.05\end{array}$ & ns & 0.02 & ns & $\begin{array}{l}- \\
0.10\end{array}$ & ns & 0.02 & ns & $\begin{array}{l}- \\
0.05\end{array}$ & ns & $\begin{array}{l}- \\
0.05\end{array}$ & ns & $\begin{array}{l}- \\
0.01\end{array}$ & ns & 0.09 & ns & 0.14 & 0.05 & 0.12 & ns \\
\hline
\end{tabular}


Table 3 Correlations between TAR/TLR and cardiometabolic variables, and between TAR/TLR and potential confounders (Continued)

\begin{tabular}{|c|c|c|c|c|c|c|c|c|c|c|c|c|c|c|c|c|c|c|c|c|}
\hline & \multicolumn{2}{|l|}{ Age } & \multicolumn{2}{|c|}{ Height } & \multicolumn{2}{|c|}{$\begin{array}{l}\text { MET- } \\
\text { minutes } \\
\text { /week }\end{array}$} & \multicolumn{2}{|c|}{$\begin{array}{l}\text { Current } \\
\text { smoker }\end{array}$} & \multicolumn{2}{|c|}{$\begin{array}{l}\text { Alcohol } \\
\text { intake }\end{array}$} & \multicolumn{2}{|c|}{$\begin{array}{l}\text { Energy } \\
\text { intake }\end{array}$} & \multicolumn{2}{|c|}{$\begin{array}{l}\mathrm{NaCl} \\
\text { intake }\end{array}$} & \multicolumn{2}{|c|}{$\begin{array}{l}\text { Whole- } \\
\text { body fat }\end{array}$} & \multicolumn{2}{|l|}{ TAR } & \multicolumn{2}{|l|}{ TLR } \\
\hline & $r$ & $P$ & $r$ & $P$ & $r$ & $P$ & $r$ & $P$ & $r$ & $P$ & $r$ & $P$ & $r$ & $P$ & $r$ & $P$ & $r$ & $P$ & $r$ & $P$ \\
\hline HOMA-IR & $\begin{array}{l}-13 \\
0.13\end{array}$ & ns & 0.05 & ns & $\begin{array}{l}- \\
0.14\end{array}$ & 0.05 & 0.02 & ns & $\overline{0}-13$ & ns & $\overline{0}-05$ & ns & $\begin{array}{l}- \\
0.09\end{array}$ & ns & 0.41 & $\begin{array}{l}< \\
0.01\end{array}$ & 0.36 & $\begin{array}{l}< \\
0.01\end{array}$ & 0.32 & $\begin{array}{l}\overline{<} \\
0.01\end{array}$ \\
\hline $\begin{array}{l}\text { Aspartate } \\
\text { aminotransferase }\end{array}$ & 0.11 & ns & $\overline{-}-$ & ns & 0.02 & ns & - & 0.04 & 0.13 & ns & - & ns & $\begin{array}{c}- \\
0.01\end{array}$ & ns & $-\overline{0}$ & ns & 0.07 & ns & 0.08 & ns \\
\hline Alanine aminotransferase & $\overline{-}-$ & ns & $\overline{-} \cdot$ & ns & $\overline{-}-$ & ns & $\overline{-} .06$ & ns & $\overline{-}-$ & ns & 0.02 & ns & $-\overline{12}$ & ns & 0.11 & ns & 0.23 & $\begin{array}{c}< \\
0.01\end{array}$ & 0.23 & $\begin{array}{l}< \\
0.01\end{array}$ \\
\hline $\begin{array}{l}\text { Estimated glomerular } \\
\text { filtration rate }\end{array}$ & $\overline{-}-$ & ns & $\overline{-}-$ & ns & 0.07 & ns & 0.00 & ns & 0.09 & ns & 0.08 & ns & 0.00 & ns & 0.05 & ns & $\overline{0.17}$ & 0.02 & $-\overline{1}$ & 0.01 \\
\hline TAR & $\overline{0} .15$ & ns & 0.02 & ns & $\overline{0} .06$ & ns & 0.03 & ns & 0.04 & ns & $\overline{0} .09$ & ns & 0.00 & ns & 0.51 & $\begin{array}{l}< \\
0.01\end{array}$ & N/A & & N/A & \\
\hline TLR & $\begin{array}{l}- \\
0.15\end{array}$ & 0.03 & 0.02 & ns & $\begin{array}{l}- \\
0.05\end{array}$ & ns & 0.01 & ns & 0.04 & ns & $\begin{array}{l}- \\
0.10\end{array}$ & ns & 0.01 & ns & 0.47 & $\begin{array}{l}< \\
0.01\end{array}$ & N/A & & N/A & \\
\hline
\end{tabular}

Biochemical marker values were logarithmically converted and then statistically analyzed Pearson's correlation was used. $P<0.05$ was considered statistically significant MET-minutes/week was calculated using the International Physical Activity Questionnaire

MET, metabolic equivalent of task; N, number; ns, not significant; TAR, trunk-to-appendicular fat ratio; TLR, trunk-to-leg fat ratio; HDL, high-density lipoprotein; LDL, low-density lipoprotein; HOMA-IR, homeostasis model assessment-insulin resistance; N/A, not applicable

before statistical analyses, analyzed, and expressed as geometric means. Medication status might affect cardiometabolic variables. Thus, subjects were stratified by mediation status to evaluate the association between TAR/ TLR and cardiometabolic variables. Comparisons between users of hypertension, dyslipidemia, or diabetes medication ("user group") and those who did not use such medication ("non-user group") were performed using the unpaired $t$ test or Mann-Whitney $U$ test. Correlations among cardiometabolic variables, potential confounders, and TAR/TLR were evaluated using Pearson's correlation coefficients. Associations between TAR/TLR and cardiometabolic variables in the non-user or user group were evaluated using multiple regression analysis after adjusting for age, height, MET-minutes/ week, current smoking, alcohol intake, energy intake, $\mathrm{NaCl}$ intake, and whole-body fat.

\section{Results}

Table 1 shows the characteristics of men who used $\geq 1$ medication for hypertension, dyslipidemia, or diabetes (user group) and those who did not use any medication (non-user group). Both TAR and TLR in the user group were significantly larger compared to those in the nonuser group. Table 2 shows differences between the user and non-user groups according to the type of medication. Both TAR and TLR for each type of medication were significantly larger in the user group compared to the non-user group.

Table 3 shows correlations between TAR/TLR and cardiometabolic variables, and between TAR/TLR and potential confounders. In the non-user group $(N=250)$, both TAR and TLR were significantly correlated with LDL cholesterol, HDL cholesterol, triglyceride, hemoglobin A1c, fasting serum insulin, fasting plasma glucose, and HOMA-IR. Similarly, both TAR and TLR in nonoverweight men of the non-user group $(N=199)$ were significantly correlated with LDL cholesterol, HDL cholesterol, triglyceride, fasting serum insulin, and HOMA-IR. Both TAR and TLR also showed significant positive correlations with whole-body fat in the non-user group and non-overweight men of the non-user group.

Table 4 shows associations between TAR/TLR and cardiometabolic variables after adjusting for potential confounders including whole-body fat. Even after adjusting for age, height, MET-minutes/week, current smoking, alcohol intake, energy intake, $\mathrm{NaCl}$ intake, and whole-body fat, both TAR and TLR were significantly associated with LDL/HDL cholesterol, triglyceride, fasting serum insulin, and HOMA-IR in the non-user group and non-overweight men of the non-user group.

Table 5 shows associations between TAR/TLR and cardiometabolic variables after adjusting for potential confounders including whole-body fat according to the type of medication. There were significant inverse associations between TAR/TLR and HDL cholesterol for each type of medication in the non-user group. In addition, there were significant positive associations between TAR/TLR and LDL cholesterol, triglyceride, hemoglobin A1c, fasting serum insulin, fasting plasma glucose, and HOMA-IR for each type of medication in the non-user group.

\section{Discussion}

The present cross-sectional study showed significant associations between trunk-to-peripheral fat ratio and 
Table 4 Associations between TAR/TLR and cardiometabolic variables after adjusting for potential confounders

\begin{tabular}{|c|c|c|c|c|c|c|c|c|}
\hline & \multirow{2}{*}{\multicolumn{2}{|c|}{$\begin{array}{l}\text { Total subjects, } \\
N=597\end{array}$}} & \multicolumn{6}{|c|}{ Medications for hypertension, dyslipidemia, or diabetes } \\
\hline & & & \multicolumn{2}{|c|}{ Users, $N=347$} & \multicolumn{2}{|c|}{ Non-users, $N=250$} & \multicolumn{2}{|c|}{ Non-overweight non-users, $N=199$} \\
\hline & Beta & $P$ & Beta & $P$ & Beta & $P$ & Beta & $P$ \\
\hline \multicolumn{9}{|l|}{ TAR } \\
\hline Systolic blood pressure & 0.03 & ns & 0.04 & ns & 0.03 & ns & -0.02 & ns \\
\hline Diastolic blood pressure & 0.00 & ns & 0.04 & ns & -0.02 & ns & -0.09 & ns \\
\hline LDL cholesterol & 0.09 & 0.04 & 0.04 & ns & 0.19 & $<0.01$ & 0.22 & $<0.01$ \\
\hline HDL cholesterol & -0.25 & $<0.01$ & -0.25 & $<0.01$ & -0.22 & $<0.01$ & -0.20 & $<0.01$ \\
\hline Total cholesterol & 0.02 & ns & -0.02 & ns & 0.13 & ns & 0.16 & ns \\
\hline Triglyceride & 0.32 & $<0.01$ & 0.33 & $<0.01$ & 0.28 & $<0.01$ & 0.27 & $<0.01$ \\
\hline Hemoglobin A1c & 0.17 & $<0.01$ & 0.12 & 0.04 & 0.18 & 0.02 & 0.15 & ns \\
\hline Fasting serum insulin & 0.25 & $<0.01$ & 0.23 & $<0.01$ & 0.23 & $<0.01$ & 0.19 & $<0.01$ \\
\hline Fasting plasma glucose & 0.17 & $<0.01$ & 0.12 & 0.03 & 0.17 & 0.02 & 0.12 & ns \\
\hline HOMA-IR & 0.25 & $<0.01$ & 0.23 & $<0.01$ & 0.25 & $<0.01$ & 0.20 & $<0.01$ \\
\hline \multicolumn{9}{|l|}{ TLR } \\
\hline Systolic blood pressure & 0.03 & ns & 0.04 & ns & 0.03 & ns & -0.02 & ns \\
\hline Diastolic blood pressure & -0.01 & ns & 0.03 & ns & -0.03 & ns & -0.10 & ns \\
\hline LDL cholesterol & 0.08 & ns & 0.02 & ns & 0.20 & $<0.01$ & 0.23 & $<0.01$ \\
\hline HDL cholesterol & -0.24 & $<0.01$ & -0.25 & $<0.01$ & -0.20 & $<0.01$ & -0.18 & 0.01 \\
\hline Total cholesterol & 0.02 & ns & -0.03 & ns & 0.16 & 0.03 & 0.18 & 0.03 \\
\hline Triglyceride & 0.32 & $<0.01$ & 0.32 & $<0.01$ & 0.29 & $<0.01$ & 0.28 & $<0.01$ \\
\hline Hemoglobin A1c & 0.17 & $<0.01$ & 0.12 & 0.03 & 0.19 & $<0.01$ & 0.15 & ns \\
\hline Fasting serum insulin & 0.25 & $<0.01$ & 0.24 & $<0.01$ & 0.21 & $<0.01$ & 0.16 & 0.03 \\
\hline Fasting plasma glucose & 0.17 & $<0.01$ & 0.12 & 0.03 & 0.17 & 0.02 & 0.09 & ns \\
\hline HOMA- IR & 0.25 & $<0.01$ & 0.24 & $<0.01$ & 0.23 & $<0.01$ & 0.16 & 0.03 \\
\hline
\end{tabular}

Biochemical marker values were logarithmically converted and then statistically analyzed

Multiple regression analysis was used after adjusting for age, height, MET-minutes/week, current smoker, alcohol intake, energy intake, NaCl intake, and whole-body fat

Beta represents the standard coefficient

$P<0.05$ was considered statistically significant

MET-minutes/week was calculated using the International Physical Activity Questionnaire

$N$, number; ns, not significant; TAR, trunk-to-appendicular fat ratio; TLR, trunk-to-leg fat ratio; HDL, high-density lipoprotein; LDL, low-density lipoprotein; HOMA-IR, homeostasis model assessment-insulin resistance; MET, metabolic equivalent of task

cardiometabolic variables (LDL cholesterol, HDL cholesterol, triglyceride, hemoglobin A1c, fasting serum insulin, fasting plasma glucose, and HOMA-IR) in elderly Japanese men who did not use hypertension, dyslipidemia, or diabetes medication. These associations remained significant even after whole-body fat was accounted for, indicating that trunk-to-peripheral fat ratio, independently of overall fat mass, was associated with cardiometabolic variables. In addition, these associations were also observed among non-overweight participants. These results indicate that a large volume of body fat may not be a prerequisite for the development of cardiometabolic abnormalities, and weight-independent indices (non-weight-reliant indices) such as regional fat ratios may provide additional information for the evaluation of health risks.

A previous cross-sectional study from a representative Japanese female population (the JPOS study) reported that TAR was significantly correlated with LDL cholesterol, HDL cholesterol, and hemoglobin A1c levels and that the relationships between fat ratio and cardiometabolic variables were independent of relationships between fat volume and cardiometabolic variables [18]. Our present results from elderly Japanese men are consistent with results from that study. Thus, DXAmeasured fat mass-independent indices (e.g., TAR and TLR) may be useful for assessing cardiometabolic disease risk, particularly in underweight to normal-weight populations. Trunk-to-peripheral fat ratio may also allow the characterization of MONW individuals, who are common in Asian population.

Although BMI is the most common parameter related to adiposity for all age groups, it reflects total fat mass rather than body fat distribution. On the other hand, WHR is a weight-independent index which more 
Table 5 Associations between TAR/TLR and cardiometabolic variables after adjusting for potential confounders according to the type of medication

\begin{tabular}{|c|c|c|c|c|c|c|c|c|c|c|c|c|}
\hline & \multicolumn{12}{|c|}{ Medication for } \\
\hline & \multicolumn{4}{|c|}{ Hypertension } & \multicolumn{4}{|c|}{ Dyslipidemia } & \multicolumn{4}{|c|}{ Diabetes } \\
\hline & \multicolumn{2}{|c|}{ User, $N=256$} & \multicolumn{2}{|c|}{ Non-user, $N=341$} & \multicolumn{2}{|c|}{ User, $N=167$} & \multicolumn{2}{|c|}{ Non-user, $N=430$} & \multicolumn{2}{|c|}{ User, $N=92$} & \multicolumn{2}{|c|}{ Non-user, $N=505$} \\
\hline & Beta & $P$ & Beta & $P$ & Beta & $P$ & Beta & $P$ & Beta & $P$ & Beta & $P$ \\
\hline \multicolumn{13}{|l|}{ TAR } \\
\hline Systolic blood pressure & -0.01 & ns & 0.05 & ns & 0.17 & 0.04 & -0.02 & ns & 0.11 & ns & 0.04 & ns \\
\hline Diastolic blood pressure & -0.01 & ns & 0.00 & ns & 0.13 & ns & -0.04 & ns & 0.00 & ns & 0.04 & ns \\
\hline LDL cholesterol & 0.01 & ns & 0.18 & $<0.01$ & 0.04 & ns & 0.13 & 0.01 & 0.10 & ns & 0.12 & 0.02 \\
\hline HDL cholesterol & -0.24 & $<0.01$ & -0.25 & $<0.01$ & -0.17 & 0.03 & -0.26 & $<0.01$ & -0.31 & $<0.01$ & -0.23 & $<0.01$ \\
\hline Total cholesterol & -0.04 & ns & 0.11 & ns & 0.05 & ns & 0.03 & ns & 0.04 & ns & 0.04 & ns \\
\hline Triglyceride & 0.28 & $<0.01$ & 0.34 & $<0.01$ & 0.34 & $<0.01$ & 0.29 & $<0.01$ & 0.39 & $<0.01$ & 0.29 & $<0.01$ \\
\hline Hemoglobin A1c & 0.17 & $<0.01$ & 0.17 & $<0.01$ & 0.12 & ns & 0.17 & $<0.01$ & 0.03 & ns & 0.16 & $<0.01$ \\
\hline Fasting serum insulin & 0.24 & $<0.01$ & 0.24 & $<0.01$ & 0.26 & $<0.01$ & 0.24 & $<0.01$ & 0.21 & ns & 0.21 & $<0.01$ \\
\hline Fasting plasma glucose & 0.16 & 0.02 & 0.16 & 0.01 & 0.17 & 0.04 & 0.15 & $<0.01$ & 0.04 & ns & 0.14 & $<0.01$ \\
\hline HOMA-IR & 0.24 & $<0.01$ & 0.25 & $<0.01$ & 0.27 & $<0.01$ & 0.24 & $<0.01$ & 0.18 & ns & 0.22 & $<0.01$ \\
\hline \multicolumn{13}{|l|}{ TLR } \\
\hline Systolic blood pressure & -0.01 & ns & 0.06 & ns & 0.18 & 0.02 & -0.02 & ns & 0.11 & ns & 0.04 & ns \\
\hline Diastolic blood pressure & -0.02 & ns & 0.00 & ns & 0.13 & ns & -0.05 & ns & -0.01 & ns & 0.03 & ns \\
\hline LDL cholesterol & 0.00 & ns & 0.19 & $<0.01$ & 0.02 & ns & 0.13 & 0.01 & 0.10 & ns & 0.11 & 0.03 \\
\hline HDL cholesterol & -0.23 & $<0.01$ & -0.24 & $<0.01$ & -0.17 & 0.03 & -0.25 & $<0.01$ & -0.32 & $<0.01$ & -0.22 & $<0.01$ \\
\hline Total cholesterol & -0.05 & ns & 0.13 & 0.04 & 0.02 & ns & 0.05 & ns & 0.03 & ns & 0.05 & ns \\
\hline Triglyceride & 0.27 & $<0.01$ & 0.35 & $<0.01$ & 0.33 & $<0.01$ & 0.30 & $<0.01$ & 0.40 & $<0.01$ & 0.29 & $<0.01$ \\
\hline Hemoglobin A1c & 0.17 & $<0.01$ & 0.17 & $<0.01$ & 0.14 & ns & 0.17 & $<0.01$ & 0.04 & ns & 0.16 & $<0.01$ \\
\hline Fasting serum insulin & 0.26 & $<0.01$ & 0.22 & $<0.01$ & 0.28 & $<0.01$ & 0.23 & $<0.01$ & 0.21 & 0.05 & 0.21 & $<0.01$ \\
\hline Fasting plasma glucose & 0.17 & $<0.01$ & 0.14 & 0.02 & 0.18 & 0.03 & 0.15 & $<0.01$ & 0.04 & ns & 0.14 & $<0.01$ \\
\hline HOMA-IR & 0.26 & $<0.01$ & 0.23 & $<0.01$ & 0.28 & $<0.01$ & 0.24 & $<0.01$ & 0.19 & ns & 0.22 & $<0.01$ \\
\hline
\end{tabular}

Biochemical marker values were logarithmically converted and then statistically analyzed

Multiple regression analysis was used after adjusting for age, height, MET-minutes/week, current smoker, alcohol intake,

energy intake, $\mathrm{NaCl}$ intake, and whole-body fat

Beta represents the standard coefficient

$P<0.05$ was considered statistically significant

MET-minutes/week was calculated using the International Physical Activity Questionnaire

$N$, number; ns, not significant; TAR, trunk-to-appendicular fat ratio; TLR, trunk-to-leg fat ratio; HDL, high-density lipoprotein; LDL, low-density lipoprotein; HOMA-IR, homeostasis model assessment-insulin resistance; MET, metabolic equivalent of task

accurately describes abdominal fat distribution than BMI $[8,26,27]$. WHR is reportedly an alternative anthropometric index of obesity that overcomes the limitations of BMI, and predicts the clustering of cardiovascular risk factors in addition to weight-based indices such as BMI [28-30]. Previous studies in normal-weight populations found that high WHR levels were associated with insulin resistance markers and cardiovascular disease risk factors $[31,32]$. Therefore, WHR might be a more appropriate index for evaluating cardiometabolic disease risk in Asian people who have lower BMI levels than those of European descent [6]. However, WHR cannot distinguish between fat mass and lean mass and can only serve as a surrogate for the distribution of detrimental visceral fat, beneficial gluteal fat, and muscle [27]. On the other hand, DXA devices are capable of measuring body composition (fat mass, lean mass, and bone mass) of the entire body or specific body regions (arm, leg, and trunk) with high precision, low scanning time, and low radiation exposure [33]. DXA-measured regional fat mass can be used to assess fat distribution using the trunk-to-peripheral fat ratio. DXA-measured trunk fat consists of visceral and subcutaneous fat, while arm or leg fat does not include visceral fat. Therefore, TAR and TLR reveal the relative amount of visceral adipose tissue as weight-independent indices. Body composition-based 
DXA-measured fat ratio is a more precise method for measuring the distribution of body fat than conventional anthropometric indices such as WHR.

Although the pathophysiological background underlying the associations between trunk-to-peripheral fat ratio and cardiometabolic variables was not explored in the present study, the associations might be attributed to differences between visceral and subcutaneous adipose tissue. Subcutaneous adipose tissue acts as a "protective metabolic sink," metabolizing and storing excess free fatty acids and storing the bulk of body energy [34]. When the extra energy is channeled into subcutaneous adipose tissue, individuals with a positive energy balance would be protected against the development of metabolic syndrome. However, in individuals who lack subcutaneous adipose tissue and thus have a limited ability to store excess energy, surplus triglycerides are deposited at undesirable sites (e.g., visceral adipose tissue), a phenomenon referred to as ectopic fat deposition [34]. Given the unfavorable effects of ectopic fat deposits, the presence of subcutaneous adipose tissue may contribute to a reduced risk of various cardiometabolic outcomes by curbing ectopic fat deposition. Thus, the ratio of trunk fat (including visceral and subcutaneous fat) to peripheral fat (including subcutaneous fat) may be useful for evaluating cardiometabolic disease risk.

This study has several strengths. First, DXA can be used to determine the bone and soft tissue composition of the whole body and subregions such as the arms, legs, and the trunk and is a simple and safe technique that can be used for children and the old and frail [10]. Furthermore, the precision of all DXA measurements has been reported to be excellent [10]. Therefore, DXA is increasingly being used as a criterion or reference for comparison with other body composition measurements, such as the impedance method [35]. However, DXA-based body fat measurement is expensive and not suitable for assessing cardiovascular risk of very common lifestyle-related diseases. Second, this is the first report on relationships between trunk-toperipheral fat ratio and cardiometabolic risk factors among Japanese elderly men who tend to have more visceral adiposity than European people. Third, the FORMEN study had a relatively large sample size. Fourth, the study was conducted as part of an ongoing cohort study, allowing for further follow-up studies with the same study participants. Finally, this was a single-center study, and thus, there was no inter-center variation.

There are also some limitations worth noting. First, participants were not a random sample, but rather volunteers living in a certain region of Japan, and thus may not be representative of the entire Japanese population. Older adults who volunteer and engage in more volunteer activities have been reported to be more likely to have higher levels of well-being, including self-rated health, functional dependency, and depressive symptomatology [36]. Individuals with serious diseases may also have been less likely to participate in the present study. Second, the cross-sectional design did not allow us to determine causal relationships between predictors and outcomes.

\section{Conclusion}

The trunk-to-peripheral fat ratio, a body massindependent index, was associated with cardiometabolic variables in elderly Japanese men. Moreover, these associations were also observed among non-overweight men and were independent of associations between wholebody fat and cardiometabolic variables. Fat ratio parameters may be useful for assessing cardiometabolic risk factors, particularly in underweight to normal-weight populations. The trunk-to-peripheral fat ratio may also allow for the characterization of MONW individuals who are very common in Asian populations.

\section{Abbreviations}

BMl: Body mass index; DXA: Dual-energy X-ray absorptiometry; FFQ: Food frequency questionnaire; FFQPOP: FFQ for the Prevention and Management of Osteoporosis; FORMEN: Fujiwara-kyo Osteoporosis Risk in Men; HDL: Highdensity lipoprotein; HOMA-IR: Homeostasis model assessment-insulin resistance; IPAQ: International Physical Activity Questionnaire; JPOS: Japanese

Population-Based Osteoporosis; LDL: Low-density lipoprotein; MET: Metabolic equivalent of task; MONW: Metabolically obese, normal-weight; TAR: Trunkto-appendicular fat ratio; TLR: Trunk-to-leg fat ratio; WHR: Waist-to-hip ratio

\section{Acknowledgements}

This study represents a part of the research conducted by the second Fujiwara-kyo Osteoporosis Risk in Men study group.

\section{Authors' contributions}

KK, MI, and YF did the study conception and design. KK, YF, KO, TT, JT, AY, $J M, E K, K U$, and $\mathrm{Ml}$ contributed in the acquisition of the data. KK, MI, and YF analyzed and interpreted the data. KK, YF, KO, TT, JT, AY, JM, EK, KU, and MI drafted the manuscript or revised it critically for important intellectual content. KK wrote the paper. The authors read and approved the final version of the manuscript.

\section{Funding}

This work was supported by Grants-in-Aid for Scientific Research (JSPS KAKE NHI Grant Numbers JP15H02526, JP17K09141, JP18H03059, JP18H03109, JP18K10077, JP18K19711, JP19H04040) from the Japan Society for the Promotion of Science. The funding bodies played no role in designing the study; collecting, analyzing, and interpreting the data; writing the manuscript; or deciding on where to submit the manuscript for publication.

\section{Availability of data and materials}

The datasets generated during the current study are not publicly available, but are available from the corresponding author on reasonable request.

\section{Declarations}

\section{Ethics approval and consent to participate}

The protocol of the FORMEN study was approved by the ethics committees of Kindai University Faculty of Medicine and Kansai Medical University. The study was conducted in accordance with the ethical principles stated in the Declaration of Helsinki. Participants received printed information regarding study procedures, including radiation exposure from DXA, and provided written consent prior to the surveys. Participants were also allowed to decline participation on their own accord at any time, even during the surveys. 


\section{Consent for publication}

Not applicable.

\section{Competing interests}

The authors declare that they have no competing interests.

\section{Author details}

${ }^{1}$ Department of Hygiene and Public Health, Kansai Medical University, 2-5-1 Shin-machi, Hirakata, Osaka 573-1010, Japan. ²Department of Public Health, Kindai University Faculty of Medicine, 377-2 Oono-higashi, Osaka-Sayama, Osaka 589-8511, Japan. ${ }^{3}$ Chukyo Gakuin University Faculty of Nursing, 2216 Tokicho, Mizunami, Gifu 509-6192, Japan. ${ }^{4}$ Department of Hygiene and Public Health, Osaka Medical College, 2-7 Daigakumachi, Takatsuki, Osaka 569-8686, Japan. 5 Department of Nursing, Kio University, 4-2-2 Umami-naka, Koryo-cho, Nara 635-0832, Japan. ' 'Laboratory of Physiological Nutrition, Kagawa Nutrition University, 3-9-21 Chiyoda, Sakado, Saitama 350-0288, Japan.

Received: 26 October 2020 Accepted: 11 March 2021 Published online: 20 March 2021

\section{References}

1. Ruderman NB, Schneider SH, Berchtold P. The "metabolically-obese, normal-weight individual. Am J Clin Nutr. 1981;34(8):1617-21. https://doi. org/10.1093/ajcn/34.8.1617

2. Ruderman N, Chisholm D, Pi-Sunyer $X$, Schneider S. The metabolically obese, normal-weight individual revisited. Diabetes. 1998;47(5):699-713. https://doi.org/10.2337/diabetes.47.5.699.

3. Zhang Y, Fu J, Yang S, Yang M, Liu A, Wang L, Cao S, Sun X, Wang F, Liu D. Prevalence of metabolically obese but normal weight (MONW) and metabolically healthy but obese (MHO) in Chinese Beijing urban subjects. Biosci Trends. 2017;11(4):418-26. https://doi.org/10.5582/bst.2017.01016.

4. Tchernof A, Despres JP. Pathophysiology of human visceral obesity: an update. Physiol Rev. 2013;93(1):359-404. https://doi.org/10.1152/physrev. 00033.2011.

5. Huxley R, James WP, Barzi F, Patel JV, Lear SA, Suriyawongpaisal P, et al. Ethnic comparisons of the cross-sectional relationships between measures of body size with diabetes and hypertension. Obes Rev. 2008;9(Suppl 1):5361. https://doi.org/10.1111/j.1467-789X.2007.00439.x.

6. Yoon KH, Lee JH, Kim JW, Cho JH, Choi YH, Ko SH, Zimmet P, Son HY. Epidemic obesity and type 2 diabetes in Asia. Lancet. 2006;368(9548):16818. https://doi.org/10.1016/S0140-6736(06)69703-1.

7. Ma RC, Chan JC. Type 2 diabetes in East Asians: similarities and differences with populations in Europe and the United States. Ann N Y Acad Sci. 2013; 1281(1):64-91. https://doi.org/10.1111/nyas.12098.

8. Bjorntorp P. Abdominal fat distribution and the metabolic syndrome. J Cardiovasc Pharmacol. 1992;20(Suppl 8):S26-8. https://doi.org/10.1097/ 00005344-199200208-00006.

9. Ackerman KE, Davis B, Jacoby L, Misra M. DXA surrogates for visceral fat are inversely associated with bone density measures in adolescent athletes with menstrual dysfunction. J Pediatr Endocrinol Metab. 2011;24(7-8):497-504. https://doi.org/10.1515/jpem.2011.208.

10. Laskey MA. Dual-energy X-ray absorptiometry and body composition. Nutrition. 1996;12(1):45-51. https://doi.org/10.1016/0899-9007(95)00017-8.

11. Morimoto Y, Maskarinec G, Conroy SM, Lim U, Shepherd J, Novotny R. Asian ethnicity is associated with a higher trunk/peripheral fat ratio in women and adolescent girls. J Epidemiol. 2012;22(2):130-5. https://doi.org/10.2188/ jea.JE20110100.

12. Peppa M, Koliaki C, Hadjidakis DI, Garoflos E, Papaefstathiou A, Katsilambros N, Raptis SA, Dimitriadis GD. Regional fat distribution and cardiometabolic risk in healthy postmenopausal women. Eur J Intern Med. 2013;24(8):824-31. https://doi.org/10.1016/j.ejim.2013.07.001.

13. Walton C, Lees B, Crook D, Worthington M, Godsland IF, Stevenson JC. Body fat distribution, rather than overall adiposity, influences serum lipids and lipoproteins in healthy men independently of age. Am J Med. 1995;99(5): 459-64. https://doi.org/10.1016/S0002-9343(99)80220-4.

14. Aasen $G$, Fagertun $H$, Tonstad $S$, Halse J. Leg fat mass as measured by dual X-ray absorptiometry (DXA) impacts insulin resistance differently in obese women versus men. Scand J Clin Lab Invest. 2009;69(2):181-9. https://doi. org/10.1080/00365510802464641.
15. Niederauer CM, Binkley TL, Specker BL. Effect of truncal adiposity on plasma lipid and lipoprotein concentrations. J Nutr Health Aging. 2006;10:154-60.

16. Daniels SR, Morrison JA, Sprecher DL, Khoury P, Kimball TR. Association of body fat distribution and cardiovascular risk factors in children and adolescents. Circulation. 1999;99(4):541-5. https://doi.org/10.1161/01.CIR. 99.4.541.

17. Kouda K, Iki M, Fujita Y, Nakamura H, Hamada M, Uenishi K, Miyake M, Nishiyama T. Trunk-to-peripheral fat ratio predicts a subsequent blood pressure in normal-weight pubertal boys: a 3-year follow-up of the Kitakata Kids Health Study. Environ Health Prev Med. 2020;25(1):41. https://doi.org/1 0.1186/s12199-020-00878-1.

18. Kouda K, Dongmei N, Tamaki J, Iki M, Tachiki T, Kajita E, Nakatani Y, Uenishi K, Kagamimori S, Kagawa Y, Yoneshima H. Relative importance of central and peripheral adiposities on cardiometabolic variables in females: a Japanese population-based study. J Clin Densitom. 2017;20(1):58-65. https:// doi.org/10.1016/j.jocd.2016.04.004.

19. Iki M, Fujita Y, Tamaki J, Kouda K, Yura A, Kadowaki E, et al. Design and baseline characteristics of a prospective cohort study for determinants of osteoporotic fracture in community-dwelling elderly Japanese men: the Fujiwara-kyo osteoporosis risk in men (FORMEN) study. BMC Musculoskelet Disord. 2009;10(1):165. https://doi.org/10.1186/1471-2474-10-165.

20. Uenishi K, Ishida H, Nakamura K. Development of a simple food frequency questionnaire to estimate intakes of calcium and other nutrients for the prevention and management of osteoporosis. J Nutr Sci Vitaminol (Tokyo). 2008;54(1):25-9. https://doi.org/10.3177/jnsv.54.25.

21. Resources Council, Science and Technology Agency, Japan. Standard tables of food composition in Japan, 5th ed. Printing Bureau, Ministry of Finance, Tokyo. 2000 (in Japanese).

22. Tomioka K, Iwamoto J, Saeki K, Okamoto N. Reliability and validity of the International Physical Activity Questionnaire (IPAQ) in elderly adults: the Fujiwara-kyo Study. J Epidemiol. 2011;21(6):459-65. https://doi.org/10.2188/ jea.JE20110003.

23. The IPAQ group. Guidelines for data processing and analysis of the International Physical Activity Questionnaire (IPAQ)-Short and long Forms 2005. http://www.ipaq.ki.se. Accessed 26 Oct 2020.

24. Matthews DR, Hosker JP, Rudenski AS, Naylor BA, Treacher DF, Turner RC. Homeostasis model assessment: insulin resistance and beta-cell function from fasting plasma glucose and insulin concentrations in man. Diabetologia. 1985;28(7):412-9. https://doi.org/10.1007/BF00280883.

25. Matsuo S, Imai E, Horio M, Yasuda Y, Tomita K, Nitta K, Yamagata K, Tomino Y, Yokoyama H, Hishida A. Revised equations for estimated GFR from serum creatinine in Japan. Am. J. Kidney Dis. 2009;53(6):982-92. https://doi.org/10.1 053/j.ajkd.2008.12.034

26. Huxley R, Mendis S, Zheleznyakov E, Reddy S, Chan J. Body mass index, waist circumference and waist:hip ratio as predictors of cardiovascular risk-a review of the literature. Eur J Clin Nutr. 2010;64(1):16-22. https://doi.org/1 0.1038/ejcn.2009.68.

27. Jayedi A, Soltani S, Zargar MS, Khan TA, Shab-Bidar S. Central fatness and risk of all cause mortality: systematic review and dose-response metaanalysis of 72 prospective cohort studies. BMJ. 2020;370:m3324.

28. Coutinho T, Goel K, Correa de Sa D, Kragelund C, Kanaya AM, Zeller M, et al. Central obesity and survival in subjects with coronary artery disease: a systematic review of the literature and collaborative analysis with individual subject data. J Am Coll Cardiol. 2011;57:1877-1886, 19, DOl: https://doi.org/1 0.1016/j.jacc.2010.11.058

29. Czernichow S, Kengne AP, Stamatakis E, Hamer M, Batty GD. Body mass index, waist circumference and waist-hip ratio: which is the better discriminator of cardiovascular disease mortality risk?: evidence from an individual-participant meta-analysis of 82864 participants from nine cohort studies. Obes Rev. 2011; 12(9):680-7. https://doi.org/10.1111/j.1467-789X.2011.00879.x.

30. Hori A, Nanri A, Sakamoto N, Kuwahara K, Nagahama S, Kato N, Fukasawa K, Nakamoto K, Ohtsu M, Matsui A, Kochi T, Eguchi M, Imai T, Nishihara A, Tomita K, Murakami T, Shimizu C, Shimizu M, Miyamoto T, Uehara A, Yamamoto M, Nakagawa T, Yamamoto S, Honda T, Okazaki H, Sasaki N, Kurotani K, Pham NM, Kabe I, Mizoue T, Sone T, Dohi S, for the Japan Epidemiology Collaboration on Occupational Health Study Group. Comparison of body mass index, waist circumference, and waist-to-height ratio for predicting the clustering of cardiometabolic risk factors by age in Japanese workers--Japan Epidemiology Collaboration on Occupational Health study. Circ J. 2014;78(5):1160-8. https://doi.org/10.1253/circj.CJ-13-1067. 
31. Pihl E, Jurimae T. Cardiovascular disease risk factors in males with normal body weight and high waist-to-hip ratio. J Cardiovasc Risk. 2001;8(5):299305. https://doi.org/10.1177/174182670100800509.

32. Benites-Zapata VA, Toro-Huamanchumo CJ, Urrunaga-Pastor D, GuarnizoPoma M, Lazaro-Alcantara H, Paico-Palacios S, Pantoja-Torres B, RanillaSeguin VC. High waist-to-hip ratio levels are associated with insulin resistance markers in normal-weight women. Diabetes Metab Syndr. 2019; 13(1):636-42. https://doi.org/10.1016/j.dsx.2018.11.043.

33. Albanese CV, Diessel E, Genant HK. Clinical applications of body composition measurements using DXA. J Clin Densitom. 2003;6(2):75-85. https://doi.org/10.1385/JCD:6:2:75.

34. Despres JP. Body fat distribution and risk of cardiovascular disease: an update. Circulation. 2012;126(10):1301-13. https://doi.org/10.1161/CIRCULA TIONAHA.111.067264.

35. Shypailo RJ, Butte NF, Ellis KJ. DXA: can it be used as a criterion reference for body fat measurements in children? Obesity (Silver Spring). 2008;16:457-62.

36. Morrow-Howell N, Hinterlong J, Rozario PA, Tang F. Effects of volunteering on the well-being of older adults. J Gerontol B Psychol Sci Soc Sci. 2003; 58(3):S137-45. https://doi.org/10.1093/geronb/58.3.S137.

\section{Publisher's Note}

Springer Nature remains neutral with regard to jurisdictional claims in published maps and institutional affiliations.

Ready to submit your research? Choose BMC and benefit from:

- fast, convenient online submission

- thorough peer review by experienced researchers in your field

- rapid publication on acceptance

- support for research data, including large and complex data types

- gold Open Access which fosters wider collaboration and increased citations

- maximum visibility for your research: over $100 \mathrm{M}$ website views per year

At BMC, research is always in progress.

Learn more biomedcentral.com/submissions 